\title{
Laser Assisted Treatment of Extra Oral Cutaneous Sinus Tract of Endodontic Origin: A Case Report
}

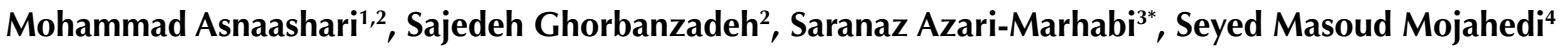 \\ ${ }^{1}$ Laser Application in Medical Sciences Research Center, Center of Excellence for Training in Medical Laser Applications, \\ Shahid Beheshti University of Medical Sciences, Tehran, Iran \\ ${ }^{2}$ Department of Endodontics, School of Dentistry, Shahid Beheshti University of Medical Sciences, Tehran, Iran \\ ${ }^{3}$ Department of Oral Medicine, School of Dentistry, Shahid Beheshti University of Medical Sciences, Tehran, Iran \\ ${ }^{4}$ Department of Laser, School of Dentistry, Shahid Beheshti University of Medical Sciences, Tehran, Iran
}

\author{
*Correspondence to \\ Saranaz Azari-Marhabi, \\ DDS; Department of Oral \\ and Maxillofacial Medicine \\ School of Dentistry, Shahid \\ Beheshti University of Medical \\ Sciences, Tehran, Iran. \\ Tel:+98 2122413897; \\ Fax:+98 2122403075; \\ Email: saranazazari@yahoo.com
}

Published online 29 August 2017

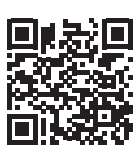

\begin{abstract}
Introduction: Sinus tract (or fistula) is a common manifestation of pulpal necrosis with periapical pathosis that requires conventional or rarely surgical- endodontic treatment in order to heal. It is mainly identified intraorally and in rare cases manifestation is extra orally, which may frequently be misdiagnosed with cutaneous lesion and incorrectly treated. New technologies such as lasers have been developed as adjuncts to standard endodontic antimicrobial procedures in order to increase the success rate of endodontic therapy while complying with the desire of saving a natural tooth.

Case Presentation: Herein, we present a clinical case of extra oral sinus tract with periodic pus drainage that was successfully treated by combining conventional endodontic therapy, intra canal photodynamic therapy (PDT), extra oral low level laser therapy (LLLT) and laser treatment for extra oral fistula.

Conclusion: Treatment of the odontogenic lesion among with the extra oral lesion was an ingenious and successful technique which was achieved via laser technology and winded up to patient's consent and satisfactory results.

Keywords: Sinus tract; Odontogenic; Photo dynamic therapy; Low level laser therapy.
\end{abstract}

\section{Introduction}

With increased patient's willingness for saving natural tooth in recent years, the standards of dental treatments have constantly been raised by utilizing new technologies. While conventional methods of endodontic therapy endeavor to eliminate bacteria within the root canal system by combining mechanical instrumentation and chemical irrigation protocols, still a large number of pathologies will occur due to our inability at eradicating bacteria from the canals of infected teeth. Even teeth may require retreatment and/or periradicular surgery in order to successfully treat persistent infections. Therefore, new technologies have been developed as adjuncts to standard endodontic antimicrobial procedures in order to increase the success rate of endodontic therapy while complying with the desire of saving a natural tooth. ${ }^{1,2}$

Abscesses, granulomas, or apical cysts are pathologies which occur due to microbial colonization of the root canal system. Formation of a sinus tract or drainage duct, called fistula is a consequence of the suppurative process of a periapical abscess. Fistula is an aberrant pathological lane between two anatomical spaces or a pathway that connects an internal cavity or organ to the surface of the body. A sinus tract is an abnormal channel that originates or ends in one opening. In the literature, the terms fistulas and sinuses are often interchangeably used. An odontogenic cutaneous fistula which is frequently misdiagnosed and incorrectly treated is a pathologic communication between the cutaneous surface of the face and the oral cavity. A cutaneous fistula with origin from dental pathology is a rare entity. In many cases patients seek treatment from a dermatologist, a family physician or a general surgeon. Since the cutaneous lesions do not always reveal the origin of the infection and only in few patients there would be a complaint of dental pain or other symptoms which complicate definitive diagnosis and often leads to misdiagnosis. Due to the misdiagnosis, they usually undergo multiple antibiotic regimens, surgical excisions and biopsies. A thorough history taking and intraoral examination are critical for early and appropriate diagnosis and could extricate the patient from unnecessary treatment. Endodontic treatment, endodontic surgery or dental extraction may eliminate the infection and lead to resolution of the lesion. . $^{3-5}$

Conventional techniques do not always suffice to predictably render root canals free of bacteria. Developing noninvasive procedures to overcome the failures of endodontic treatment would be a challenging issue for

Please cite this article as follows: Asnaashari M, Ghorbanzadeh S, Azari-Marhabi S. Laser assisted treatment of extra oral cutaneous sinus tract of endodontic origin: a case report. J Lasers Med Sci. 2017;8(Suppl 1):S68-S71. doi:10.15171/jlms.2017.s13. 
investigators in the field. Various adjuvant techniques have been proposed to overcome the problem and improve the prognosis of endodontic treatments. ${ }^{6}$

Light has been used in medicine and dentistry to improve treatment outcomes and patient's satisfactions. In endodontics lasers, have been utilized as adjuncts to raise the success of endodontic treatment and retreatments. It has been postulated in several studies that oral bacteria are sensitive to photodynamic therapy (PDT). Antimicrobial photodynamic therapy (aPDT) involves the combination of a nontoxic dye and low-intensity visible light, which produces cytotoxic reactive oxygen species in the presence of oxygen. Many biofilms are susceptible to aPDT, particularly in dental diseases. ${ }^{2,6}$

Today, low level lasers (therapeutic) are being used as treatment adjunctive devices in medicine and dentistry. They have proven to be an effective modality to expedite wound healing by means of increasing fibroblast proliferation after irradiation. ${ }^{7,8}$

Combining novel therapeutic techniques of medicine and dentistry have improved patient's satisfaction. Herein we present a case of extra oral cutaneous sinus tract of endodontic origin that was successfully treated with the combination of endodontic therapy using light for PDT and also extra orally for wound healing.

\section{Case Presentation}

A 38-year-old female with chief complaint of periodic pus drainage from a skin lesion on the face was referred to the endodontic department of Shahid Beheshti University of Medical Sciences (Figure 1). She reported history of systemic antibiotic therapy by dermatologists for treatment of the skin lesion but no sign of healing was seen in this duration. The fistula was present from 8 months ago, a few months after composite filling of tooth \#5.

Subjective finding included no pain from heat, cold and chewing and pus drainage from the fistula. Extra oral examination revealed a fibrous nodule with indurated consistency on palpation and purulent discharge from it in the zygomatic region. Findings of intra oral examination were as followed: Class $\mathrm{V}$ composite filling tooth \#5, no swelling and no mobility, normal periodontal pocket depth. Radiographic tracing with a gutta-percha cone revealed that the sinus tract led to the radiolucent area (Figure 2).

A diagnosis of necrotic pulp with chronic apical abscess of endodontic origin was established. The radiograph showed internal resorption and a large radiolucent area in the apical third of the root (Figure 3).

Treatment plan included (1)root canal therapy with $\mathrm{Ca}(\mathrm{OH}) 2$ \& photodynamic therapy, (2) low level laser therapy (LLLT) (diode laser) to improve healing of extra oral fistula, (3) composite filling, and (4) follow-up.

Also alternative treatment included extraction of first premolar and placement of implant, but the patient asked her natural tooth to be saved. After obtaining the informed consent, infiltration of local anesthesia

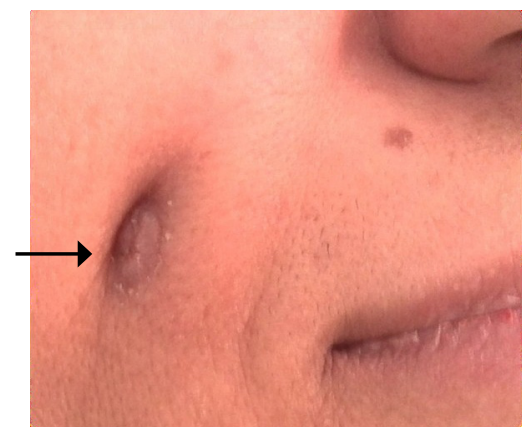

Figure 1. An Extra Oral Cutaneous Sinus Tract in the Zygomatic Region.
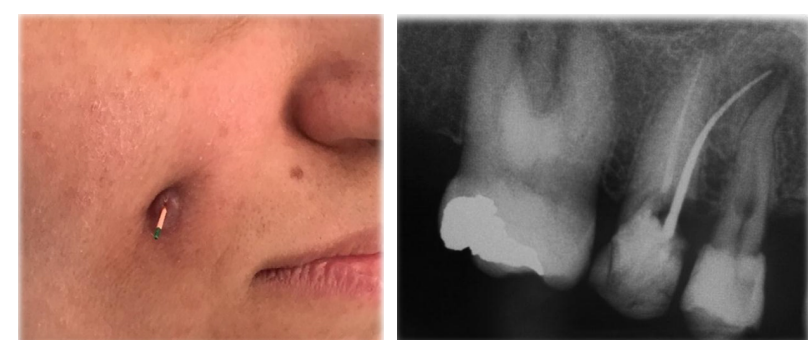

Figure 2. Radiographic Tracing With a Gutta-Percha Cone.

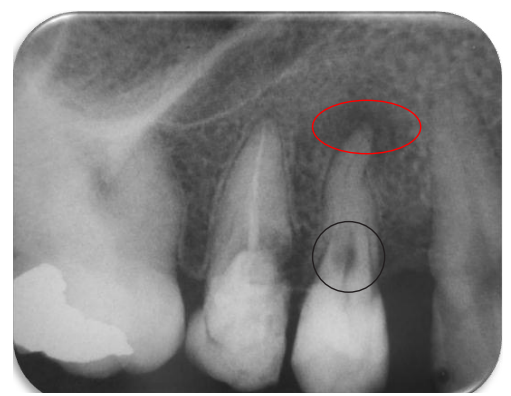

Figure 3. Internal Root Resorption and Periapical Lesion.

(lidocaine 2\% with epinephrine1/80000) was done and rubber dam was placed for isolation. Then access cavity was prepared and working length was determined. The root canal was cleaned and shaped using conventional methods. The canal was irrigated by normal saline and $\mathrm{NaOCl} 2.5 \%$ using ultrasonic devices. To improve the treatment outcomes, PDT for canal disinfection using methylene blue $(0.01 \%)$ for 5 minutes in dried canals and exposure to the appropriate wavelength of light which was light emitting diode (LED $630 \mathrm{~nm}$, FotoSan, Denmark) was performed. Then $\mathrm{Ca}(\mathrm{OH}) 2$ was placed for 2 weeks and the tooth was temporary restored. In 2 weeks the temporary restoration was removed, $\mathrm{Ca}(\mathrm{OH}) 2$ dressing was irrigated and obturation with warm vertical condensation technique (Gutta percha \& AH26) was done and the patient was referred to restorative department for composite filling (Figure 4). There was a sign of healing of the extra oral fistula in 2 weeks (Figure 5).

Since the patient was concerned and complained about 
the extra oral lesion a LLLT (diode laser, Ga-AL-As, Dr. Smile, Italy) program was planned to improve the healing of the extra oral lesion. Laser settings for low level laser program were as followed: Power: 0.3-0.4 W diode laser, Exposure duration: 30 seconds, Spectrum: Infrared (808 $\mathrm{nm}$ ) and the total sessions of LLLT: 15 sessions (2 sessions per week). Also the freckle inferior to the right nasal alar was also removed by diode laser (Ga-Al-As, $808 \mathrm{~nm}$, Dr. Smile, Italy) according to patient request (Figure 6). The patient was followed in 2 months and the treatment result was satisfactory. Six months and 12 months follow-ups also showed prosperity.

\section{Discussion}

Nowadays, innovative therapeutic techniques of medicine and dentistry have been combined to improve patient satisfaction. Hereby we presented a case of extra oral

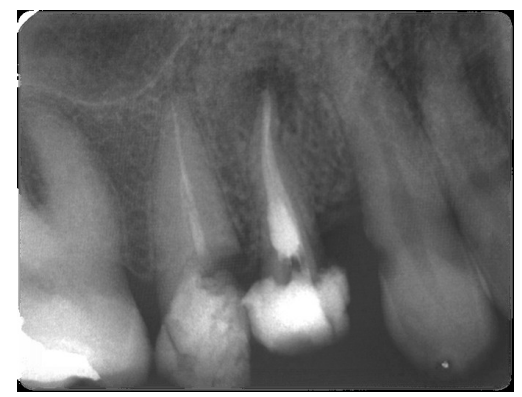

Figure 4. After Endodontic Treatment.
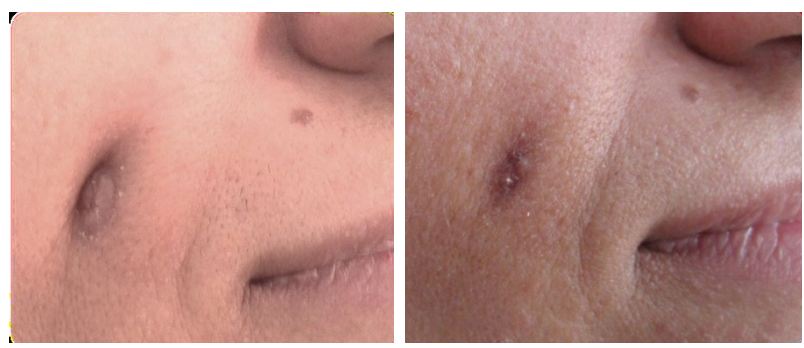

Figure 5. Healing in 2 Weeks After Endodontic Treatment. Left picture shows the extra oral fistula in the First visit and the right picture is 2 weeks after endodontic treatment.

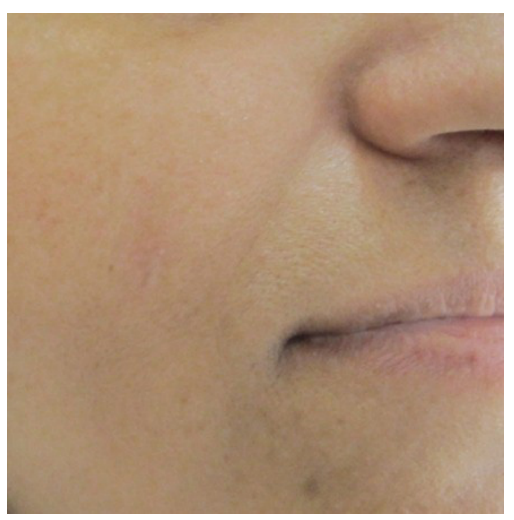

Figure 6. After Completing the Low Level Laser Therapy Procedure. cutaneous sinus tract of endodontic origin which was successfully treated with the combination of endodontic therapy and also using light intraorally for PDT and extraorally for wound healing.

Sinus tracts developing periapically from chronic low grade inflammations are considered a common clinical observation. However, atypical localization of any sinus tract's fistula extra orally can present a diagnostic challenge to the clinician. Extra oral sinus tracts of dental origin may be frequently misdiagnosed with dermatological lesions leading to incorrect treatment. Few cases of extra oral sinus tracts have been described in the literature and none of them have been treated with this innovative technique. ${ }^{3-5}$ Moreira et al reported 2 clinical cases of post-treatment apical periodontitis in which endodontic retreatment were unsuccessful despite the standard quality of the endodontic retreatment and there was persistent swelling with an intra-oral sinus tract. In these cases aPDT via sinus tract was performed and it was concluded that the protocol of aPDT associated with LPT applied via sinus tract was safe and conservative and also efficient for control of the local infection. ${ }^{9}$

Laser application in endodontics was first introduced by Weichman in 1971 and since then a variety of research on potential applications for lasers in endodontics have been done and much interest is developing in this promising field. ${ }^{10}$ Elimination of root canal bacteria is not always achievable by conventional techniques. PDT using TB and a LED lamp has the potential to be used as an adjunctive antimicrobial procedure in conventional endodontic therapy. ${ }^{6}$ In addition to $0.01 \%$ methylene blue, $630 \mathrm{~nm}$ laser wavelength was applied in the present case report, since it has been suggested that the most effective range of methylene blue solution concentration for generating singlet oxygen during the application of antimicrobial aPDT is between $0.001 \%$ and $0.01 \%$. Methylene blue is a phenothiazine dye that has maximum wavelength absorption of $656 \mathrm{~nm} .^{11,12}$

In dermatology, LLLT has been described to have beneficial effects on healing of burns, wrinkles, acne scars and hypertrophic scars. Being non-invasive and almost free of side-effects encourages further testing of LLLT in dermatology. Results of several researches have shown that LLLT could be effective in modulating both local and systemic response to improve wound healing. Obvious promotion of wound healing process using LLLT has been described by Hussein et al. ${ }^{8}$ An increase in fibroblast proliferation after the irradiation of LLLT has been shown in in-vitro studies, which suggests the facilitating power of LLLT therapy in fibroplasia during the repair phase of tissue healing. LLLT induces faster epithelialization that concludes to better formation of the epidermis. Formation of normal epidermis and disappearance of the scar have also been postulated as consequences of using LLLT. ${ }^{7,8,13}$

In accordance with the present case Asnaashari and Asnaashari also used laser technology for treatment of an extra oral fistula on the chin of a 22-year-old patient. For canal sterilization laser settings were $1 \mathrm{~W}, 2 \mathrm{~mm}$ laser per 
second in 4 times. The settings for LLLT were $4 \mathrm{~J} / \mathrm{cm}^{2}, 30$ $\mathrm{mW}$, red spectrum $(685 \mathrm{~nm})$ for the fistula and $4 \mathrm{~J} / \mathrm{cm}^{2}$, $200 \mathrm{~mW}$, infrared spectrum $(810 \mathrm{~nm})$. The total sessions of LLLT were 15 sessions ( 2 sessions per week). ${ }^{14}$

\section{Conclusion}

Dental clinicians are in an advantageous position to identify a variety of lesions and conditions with challenging diagnosis such as cutaneous fistula with endodontic origin. Treatment of the odontogenic lesion among with the extra oral lesion was an ingenious and successful technique which was achieved via laser technology and winded up to patient's consent and satisfactory results of treatment.

\section{Ethical Considerations}

Ethical principles for medical research have been applied in the process of the research.

\section{Conflict of Interests}

The authors declare that there is no conflict of interest.

\section{References}

1. Narayanan LL, Vaishnavi C. Endodontic microbiology. J Conserv Dent. 2010;13(4):233-239. doi: 10.4103/09720707.73386.

2. Asnaashari M, Mojahedi SM, Asadi Z, Azari-Marhabi S, Maleki A. A comparison of the antibacterial activity of the two methods of photodynamic therapy (using diode laser 810nm and LED lamp 630nm) against Enterococcus faecalis in extracted human anterior teeth. Photodiagnosis Photodyn Ther. 2016;13:233-237. doi:10.1016/j. pdpdt.2015.07.171.

3. Samir N, Al-Mahrezi A, Al-Sudairy S. Odontogenic cutaneous fistula: report of two cases. Sultan Qaboos Univ Med J. 2011;11(1):115-118.

4. Qazi SS, Manzoor MA, Qureshi R, Arjumand B, Hussain SM, Afridi Z. Nonsurgical endodontic management of cutaneously draining odontogenic sinus. J Ayub Med Coll Abbottabad. 2006;18(2):88-89.
5. Pasternak-Júnior B, Teixeira CS, Silva-Sousa YT, SousaNeto MD. Diagnosis and treatment of odontogenic cutaneous sinus tracts of endodontic origin: three case studies. Int Endod J. 2009;42(3):271-276.

6. Asnaashari M, Godiny M, Azari-Marhabi S, Tabatabaei FS, Barati M. Comparison of the antibacterial effect of 810 $\mathrm{nm}$ diode laser and photodynamic therapy in reducing the microbial flora of the canal during root retreatment in patients with periradicular lesions. J Lasers Med Sci. 2016;7(2):99-104. doi:10.15171/jlms.2016.17.

7. Avci P, Gupta A, Sadasivam M, et al. Low-level laser (light) therapy (LLLT) in skin: stimulating, healing, restoring. Semin Cutan Med Surg. 2013;32(1):41-52.

8. Hussein AJ, Alfars AA, Falih MAJ, Hassan ANA. Effects of a low level laser on the acceleration of wound healing in rabbits. Nh Am J Med Sci. 2011;3(4):193-197. doi:10.4297/ najms.2011.3193.

9. Moreira MSNA, de FreitasArchilla JR, Lascala CA, Ramalho K M, Gutknecht N, Marques MM. Posttreatment apical periodontitis successfully treated with antimicrobial photodynamic therapy via sinus tract and laser phototherapy: report of two cases. Photomed Laser Surg. 2015;33(10):524-528. doi:10.1089/pho.2015.3936.

10. De Moor R, Roeykens H, Meire M, Depraet F. LASER applications in endodontics. Rev Belge Med Dent (1984). 2005;60(2):115-145.

11. Komine C, Tsujimoto Y. A small amount of singlet oxygen generated via excited methylene blue by photodynamic therapy induces the sterilization of Enterococcus faecalis. J Endod. 2013;39:411-414.

12. Zand V, Milani AS, Amini M, et al. Antimicrobial efficacy of photodynamic therapy and sodium hypochlorite on monoculture biofilms of enterococcus faecalis at different stages of development. Photomed Laser Surg. 2014;32:245251.

13. Hopkins JT, McLoda TA, Seegmiller JG, David Baxter G. Low-Level Laser Therapy Facilitates Superficial Wound Healing in Humans: A Triple-Blind, Sham-Controlled Study. J Athl Train. 2004;39(3):223-229.

14. Asnaashari $M$, Asnaashari N. Clinical application of $810 \mathrm{~nm}$ diode laser and low level laser therapy for treating an endodontic problem. J Lasers Med Sci. 2011;2(2):82-86. 